\title{
Postoperative atrial fibrillation: The role of the inflammatory response
}

\author{
Yosuke Ishii, MD, PhD, ${ }^{a}$ Richard B. Schuessler, PhD, ${ }^{a}$ Sydney L. Gaynor, MD, ${ }^{b}$ Kiyomi Hames, MD,${ }^{c}$ and \\ Ralph J. Damiano, Jr, MD
}

\section{ABSTRACT}

Objective: Abnormal atrial conduction has been shown to be a substrate for postoperative atrial fibrillation (POAF). This study aimed to determine the relationship between the location of the atrial reentry responsible for POAF, and degree of atrial inflammation.

Methods: Normal mongrel dogs $(\mathrm{n}=18)$ were divided into 3 groups: anesthesia alone (anesthesia), lateral right atriotomy (atriotomy), and lateral right atriotomy with anti-inflammatory therapy (steroid). Conduction properties of the right and left atria (RA and LA) were examined 3 days postoperatively by mapping. Activation was observed during burst pacing-induced AF. The RA and LA myeloperoxidase activity was measured to quantitate the degree of inflammation.

Results: Sustained AF ( $>2$ minutes) was induced in 5 of 6 animals in the atriotomy group, but in none in the anesthesia or steroid groups. All sustained AF originated from around the RA incision. Three of these animals had an incisional reentrant tachycardia around the right atriotomy and 2 had a focal activation arising from the RA during AF. The LA activations in these animals were passive from the RA activation. The RA activation of the atriotomy group was more inhomogeneous than that of the anesthesia group (inhomogeneity index: $2.0 \pm 0.2 \mathrm{vs}$ $1.0 \pm 0.1, P<.01)$. Steroid therapy significantly normalized the RA activation after the atriotomy $(1.2 \pm 0.1, P<.01)$. The inhomogeneity of the atrial conduction correlated with the myeloperoxidase activity $(r=0.774, P<.001)$.

Conclusions: Reentrant circuits responsible for POAF are dependent on the degree of inflammation and rotate around the atriotomy. Anti-inflammatory therapy decreased the risk of postoperative AF. ( $\mathrm{J}$ Thorac Cardiovasc Surg 2017;153:1357-65)

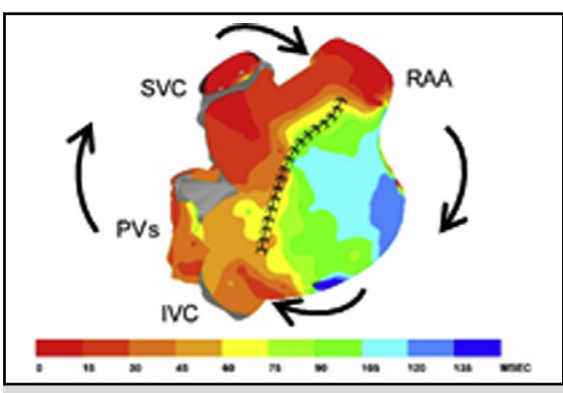

Atrial activation rotates around the right atriotomy during postoperative AF.

\section{Central Message}

Reentrant circuits responsible for postoperative AF were significantly dependent on the degree of inflammation and rotate around the atriotomy.

\section{Perspective}

POAF commonly occurs following cardiac surgery. Normal dogs were divided into 3 groups: anesthesia alone, lateral right atriotomy, and atriotomy with anti-inflammatory therapy. Reentrant circuits responsible for POAF were significantly dependent on the degree of inflammation and rotated around the atriotomy in the atriotomy group. Anti-inflammatory therapy decreased the risk of postoperative AF.

See Editorial Commentary page 1366
Postoperative atrial fibrillation (POAF) is a common complication in the perioperative phase following cardiac surgery. The incidence of POAF has been reported to be

\footnotetext{
From the a Division of Cardiothoracic Surgery, Department of Surgery, and ${ }^{\mathrm{c}}$ Department of Pathology and Immunology, Washington University School of Medicine and Barnes-Jewish Hospital, St Louis, Mo; and ${ }^{\mathrm{b}}$ Atricure, Inc, Cincinnati, Ohio. Funded by research grant R01 HL32257 from the National Institutes of Health.

Received for publication June 11, 2016; revisions received Dec 5, 2016; accepted for publication Dec 28, 2016; available ahead of print March 6, 2017.

Address for reprints: Yosuke Ishii, MD, PhD, Nippon Medical School, 1-1-5, Sendagi, Tokyo, Japan 113-8603 (E-mail: yosuke-i@nms.ac.jp).

0022-5223

Copyright (C) 2017 by The American Association for Thoracic Surgery. Published by Elsevier Inc. This is an open access article under the CC BY-NC-ND license (http:// creativecommons.org/licenses/by-nc-nd/4.0/).

http://dx.doi.org/10.1016/j.jtcvs.2016.12.051
}

approximately $25 \%$ to $30 \%$ after coronary artery bypass grafting (CABG) and $40 \%$ to $50 \%$ after valvular surgery. $^{1-3}$ This arrhythmia increases the cardiovascular mortality and morbidity, including strokes and congestive heart failure, after surgery. ${ }^{4,5}$ It has been shown that POAF significantly prolongs the postoperative length of stay and increases the average hospital costs by $50 \%$. $^{1-3,6}$

Scanning this $\mathrm{QR}$ code will take you to a supplemental video for the article.

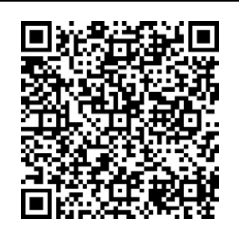




$$
\begin{aligned}
& \text { Abbreviations and Acronyms } \\
& \begin{aligned}
\text { CABG } & =\text { coronary artery bypass grafting } \\
\text { LA } & =\text { left atrium } \\
\text { NIH } & =\text { National Institutes of Health } \\
\text { OD } & =\text { optical density } \\
\text { PAF } & =\text { platelet activation factor } \\
\text { PCL } & \text { pacing cycle length } \\
\text { POAF } & =\text { postoperative atrial fibrillation } \\
\text { POD } & \text { postoperative day } \\
\text { RA } & =\text { right atrium } \\
\text { SR } & \text { sinus rhythm } \\
\text { TNF } & =\text { tumor necrosis factor }
\end{aligned}
\end{aligned}
$$

Progress in surgical techniques, anesthesia, and myocardial protection has never decreased the incidence of POAF. ${ }^{7,8}$ Occurrence of POAF after cardiac surgery is transient and self-limiting. Studies show that the peak incidence of $\mathrm{AF}$ following $\mathrm{CABG}$ is on the second postoperative day (POD) with more than one-third of such episodes occurring on that day. ${ }^{1,3}$ Of patients destined to experience POAF, in $>80 \%$ the arrhythmia terminates by the end of POD 5. ${ }^{1,3}$ Moreover, the incidence of POAF varies depending on the type of surgical procedure. . $^{2,5}$

The underlying mechanism of POAF has been described as multifactorial. Proposed causative mechanisms include atrial inflammation, ${ }^{10-13}$ excessive production of catecholamines, autonomic nervous system dysfunction, and interstitial mobilization of fluid with resultant changes in the volume, pressure, and neurohumoral environment. We have shown that atrial inflammation affects the inhomogeneity of the atrial conduction. ${ }^{14}$ We hypothesized that spatial inhomogeneity of the atrial conduction would cause POAF. The objective of this study was to determine the relationship between the location of the atrial reentry responsible for POAF, abnormalities in the atrial conduction, and degree of atrial inflammation, and to verify the effectiveness of the anti-inflammatory therapy to prevent POAF.

\section{METHOD AND MATERIALS Surgical Preparation}

All animals received humane care in compliance with the "Principles of Laboratory Animal Care," formulated by the National Society for Medical Research, and the "Guide for the Care and Use of Laboratory Animals," prepared by the National Academy of Sciences and published by the National Institutes of Health (NIH Publication 86-23, revised 1985). In addition, this study protocol was approved by the Animal Studies Committee of the Washington University School of Medicine.

Eighteen adult mongrel dogs, weighing between 25 and $30 \mathrm{~kg}$, were randomized to anesthesia alone (anesthesia group, $\mathrm{n}=6$ ), a pericardiotomy and a $5-\mathrm{cm}$ lateral right atriotomy (atriotomy group, $\mathrm{n}=6$ ), or a pericardiotomy and a 5-cm lateral right atriotomy with administration of methylprednisolone $(2 \mathrm{mg} / \mathrm{kg} / \mathrm{d})$ continuously for 1 week before the atriotomy until 3 days after the atriotomy to prevent systemic inflammation (steroid group, $\mathrm{n}=6$ ).
All animals were anesthetized with intravenous propofol $(5-7 \mathrm{mg} / \mathrm{kg})$, intubated with a cuffed endotracheal tube, and mechanically ventilated with a pressure-controlled ventilator. An adequate level of anesthesia was maintained by inhaled isoflurane (1\%-3\%). A limb-lead electrocardiogram was monitored. The total anesthesia time for each animal was standardized at 4 hours.

After an initial electrophysiological study, a median sternotomy was performed in the atriotomy and steroid groups. To mimic cardiac surgery, a lateral right atrial incision $(5 \mathrm{~cm})$ was made using the closed heart technique without the use of cardiopulmonary bypass as described previously (Figure 1, A). ${ }^{15}$ No major atrial arterial branches were divided by the atrial incision to avoid any atrial myocardial infarctions. The chest was closed in layers. The animals were injected with an intramuscular antibiotic drug (cefazolin sodium $20 \mathrm{mg} / \mathrm{kg}$ ) for 3 days postoperatively.

Three days after the initial surgery, each animal was anesthetized again with intravenous propofol $(5-7 \mathrm{mg} / \mathrm{kg})$, intubated with a cuffed endotracheal tube, and mechanically ventilated with a pressure-controlled respirator because it has been described that the peak incidence of $\mathrm{AF}$ following $\mathrm{CABG}$ is on POD 2 with more than one-third of such episodes occurring on that day. An adequate level of anesthesia was maintained by inhaled isoflurane ( $1 \%-3 \%)$. A limb-lead electrocardiogram was monitored. A median re-sternotomy was performed and the right atrium (RA) and left atrium (LA) were exposed (Figure 1, B).

\section{Electrophysiological Studies}

The RA and LA were mapped during continuous pacing and induced AF with a custom-made electrode patch containing 256 bipolar electrodes. The electrode patch was constructed from a form-fitting silicon elastomer (Specialty Silicone Fabricators Inc, Paso Robles, Calif) that fit snugly on the entire atrial epicardium and contained silver electrodes $0.5 \mathrm{~mm}$ in diameter (Pacific Wire \& Cable, Santa Ana, Calif). The interelectrode distance between the bipolar points was $5 \mathrm{~mm}$. Limb-lead electrocardiograms were simultaneously recorded. Atrial activation sequence data were recorded during spontaneous rhythm and continuous pacing at a pacing cycle length (PCL) of $300 \mathrm{~ms}$ as described previously. ${ }^{14}$ Computer-generated activation sequence maps were constructed from the recordings. Activation maps were displayed on a 3-dimensional surface model of the dog's atrium.

Atrial conduction of the RA and LA was mapped during induced AF. To initiate $\mathrm{AF}$, burst pacing from the LA at cycle lengths from 90 to $10 \mathrm{~ms}$ decremented by $10 \mathrm{~ms}$ was used. The duration of the induced $\mathrm{AF}$ was recorded for at least 3 minutes. AF was defined as an atrial activation interval of $<150 \mathrm{~ms}$, with an irregular electrogram morphology and rate.

\section{Inhomogeneity of the RA and LA Conduction}

The inhomogeneity of the atrial conduction was quantified by the variation coefficient of the maximum local activation phase difference as described previously. ${ }^{16}$ Thirty-five bipolar electrodes on the lateral RA and 20 bipolar electrodes on the LA were selected for measuring the inhomogeneity of the conduction. To evaluate the RA and LA conduction properties, a bipolar pacing electrode was placed on the right atrial appendage to initiate a wavefront that propagated parallel to the atrial incision. Continuous pacing was conducted at a cycle length of $300 \mathrm{~ms}$. The stimulus output was set at twice the pacing threshold. The activation map during continuous pacing $(\mathrm{PCL}=300 \mathrm{~ms}$ ) was constructed. The maximum phase differences were plotted as a histogram. The median $\left(\mathrm{P}_{50}\right)$ and the absolute inhomogeneity of the conduction $\left(\mathrm{P}_{5-95}\right)$ were determined from the histogram. The inhomogeneity index was calculated as a variation coefficient $\left(\mathrm{P}_{5-95} / \mathrm{P}_{50}\right) .{ }^{16}$

\section{Quantification of Atrial Inflammation}

The myeloperoxidase activities in the RA and LA myocardium were measured to quantitate the degree of inflammation. After completion of the electrophysiologic measurements, the lateral RA and LA were excised for measurement of the myeloperoxidase activity and later pathological 

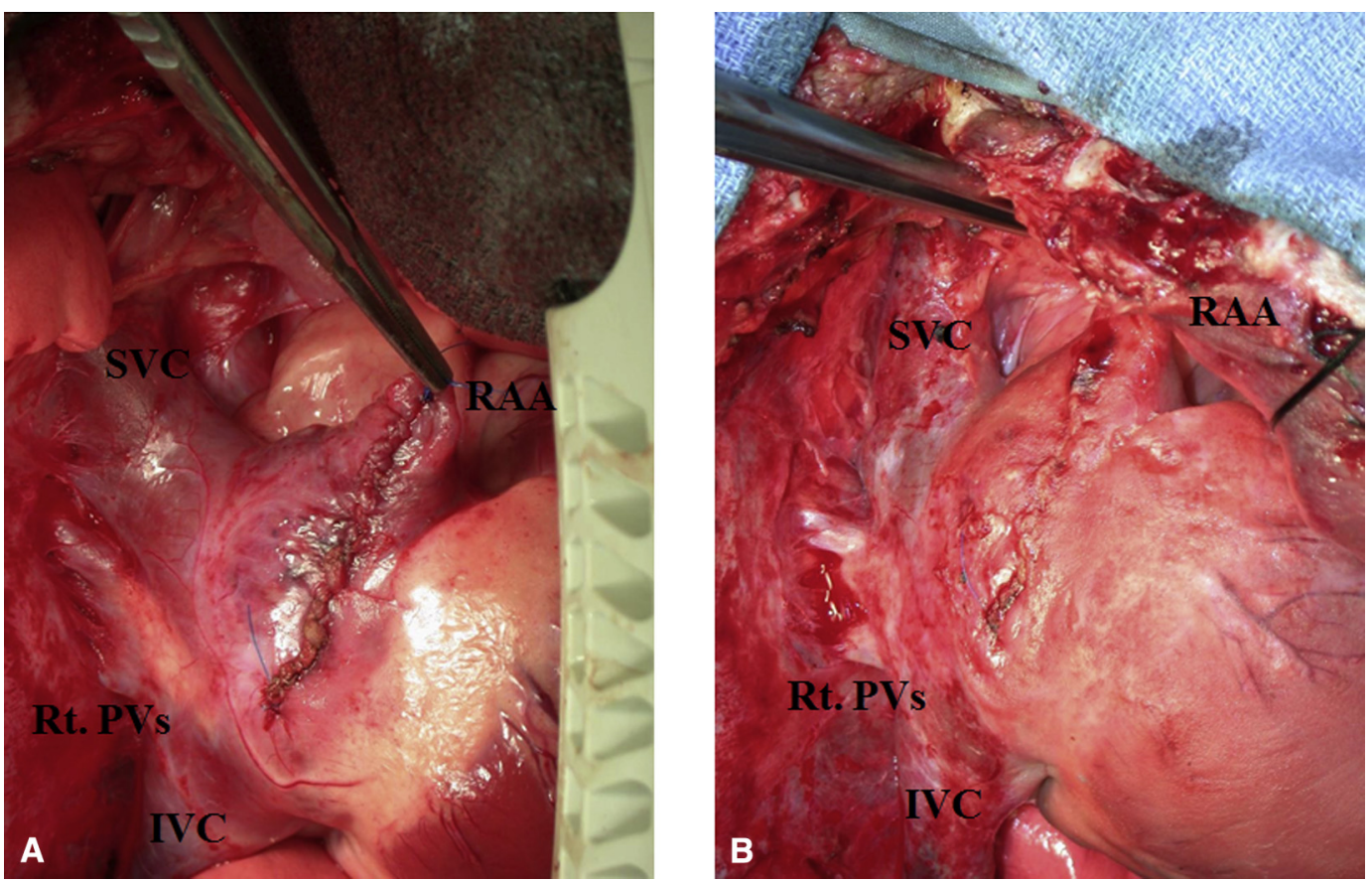

FIGURE 1. The right atriotomy during the initial surgery (A) and 3 days after the initial surgery (B). $S V C$, Superior vena cava; $R A A$, right atrial appendage; $R T P V s$, right pulmonary veins; $I V C$, inferior vena cava.

examination. The lateral RA and LA tissues were frozen in liquid nitrogen immediately after euthanasia. The quantitative myeloperoxidase activity of the atrial tissue was determined as previously described. ${ }^{17}$ The optical density (OD) was measured at $460 \mathrm{~nm}$ with a spectrophotometer (model PMQ II; Carl Zeiss, Oberkochen, Germany). Color development was linear from 5 to 20 minutes. One unit of enzyme activity was defined as 1.0 OD units per minute per milligram of tissue protein at room temperature.

\section{Pathological Examination}

The myocardial infiltration of the neutrophil cells was examined histologically. The excised lateral RA and LA tissues were fixed with $10 \%$ formalin solution and embedded in paraffin. Deparaffinized tissue sections were stained with hematoxylin-eosin. The amount of neutrophil cells in the atrial myocardium was measured microscopically. The tissue sections were observed under $\times 20$ magnification with light microscopy (BX 51; Olympus, Melville, NY) and then directly captured with a digital camera (S97809; Olympus, Melville, NY). The captured sections were converted to image files. The intensity and extent were graded from grade 0 to 4 as previously described. ${ }^{18}$ Grade 0 intensity was defined as absence of neutrophil cells in the myocardium and grade 4 was defined as dense neutrophil cell infiltration in and around the capillaries. The neutrophil cell infiltration index was calculated as the grade of the intensity $\times$ extent. All samples were graded by a histologist $(\mathrm{KY})$ blinded to the experimental groups.

\section{Statistical Analysis}

All continuous values were expressed as the mean \pm 1 standard deviation. All data were checked for normality (Shapiro-Wilk test) and equality of variance (Bartlett test). If needed, a $\log _{10}$ transform was done and the data were retested for the normality and equality of variances. Multigroup data were compared using the analysis of variance mode. Post hoc multiple comparisons were made using the Fisher least significant difference technique. Only the AF duration data failed the normality and equality of variance tests and were compared using the Kruskal-Wallis test. Correlation coefficients were calculated using the Spearman rank method. A significance level of .05 was considered statistically significant. All calculations were made using Systat version 11 software (Systat Software Inc, Richmond, Calif).

\section{RESULTS}

\section{Electrophysiological Findings}

Induction of AF during burst pacing. AF could be induced in the animals $(50 \%$ in the anesthesia group, $100 \%$ in the atriotomy group, and $33 \%$ in the steroid group) by burst pacing from the LA. All AF was induced multiple times. Only the atriotomy group $(n=5 / 6)$ had sustained AF of more than 2 minutes following burst pacing, and this was the only group in which more than half of the animals could be induced into any AF. The duration of induced AF in the atriotomy group (148 \pm 54 seconds) was significantly longer than that in the anesthesia group $(40 \pm 45$ seconds, $P<.01)$. Anti-inflammatory therapy significantly shortened the duration of induced AF $(4 \pm 6$ seconds, $P<.001)$ compared with that in the atriotomy group.

The medians (interquartile range) of the duration of the induced AF were 30 (90) in the anesthesia group, 180 (6) in the atriotomy group, and $0(10)$ in the steroid group $(P=.004$; Kruskal-Wallis test). Multiple comparisons between groups were performed with the Dwass-SteelChritchlow-Fligner test. The median of the duration of the anesthesia group was significantly different compared with that of the atriotomy group $(P=.002)$. That of the atriotomy group was also significantly different from that of the steroid group $(P<.001)$. Those of the anesthesia and steroid groups were not different $(P=.205)$. 


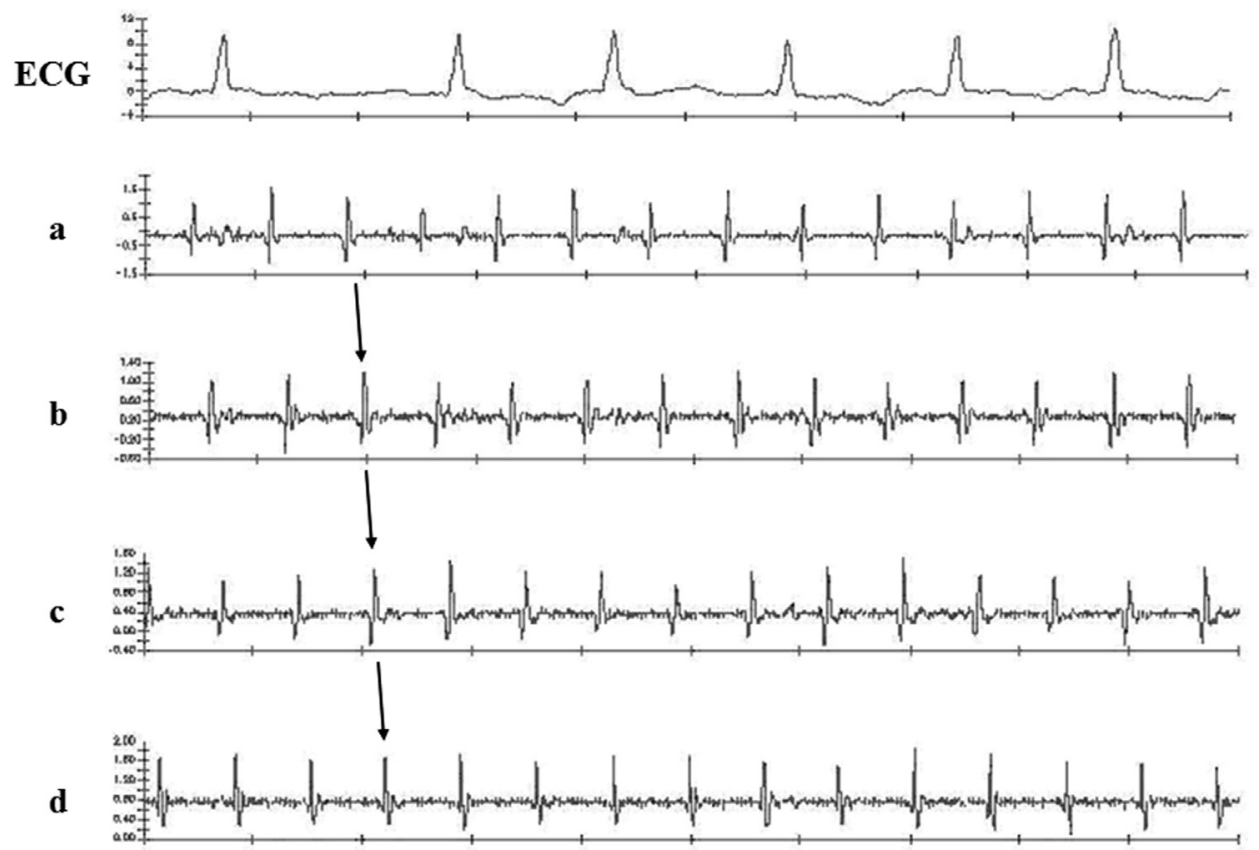

A

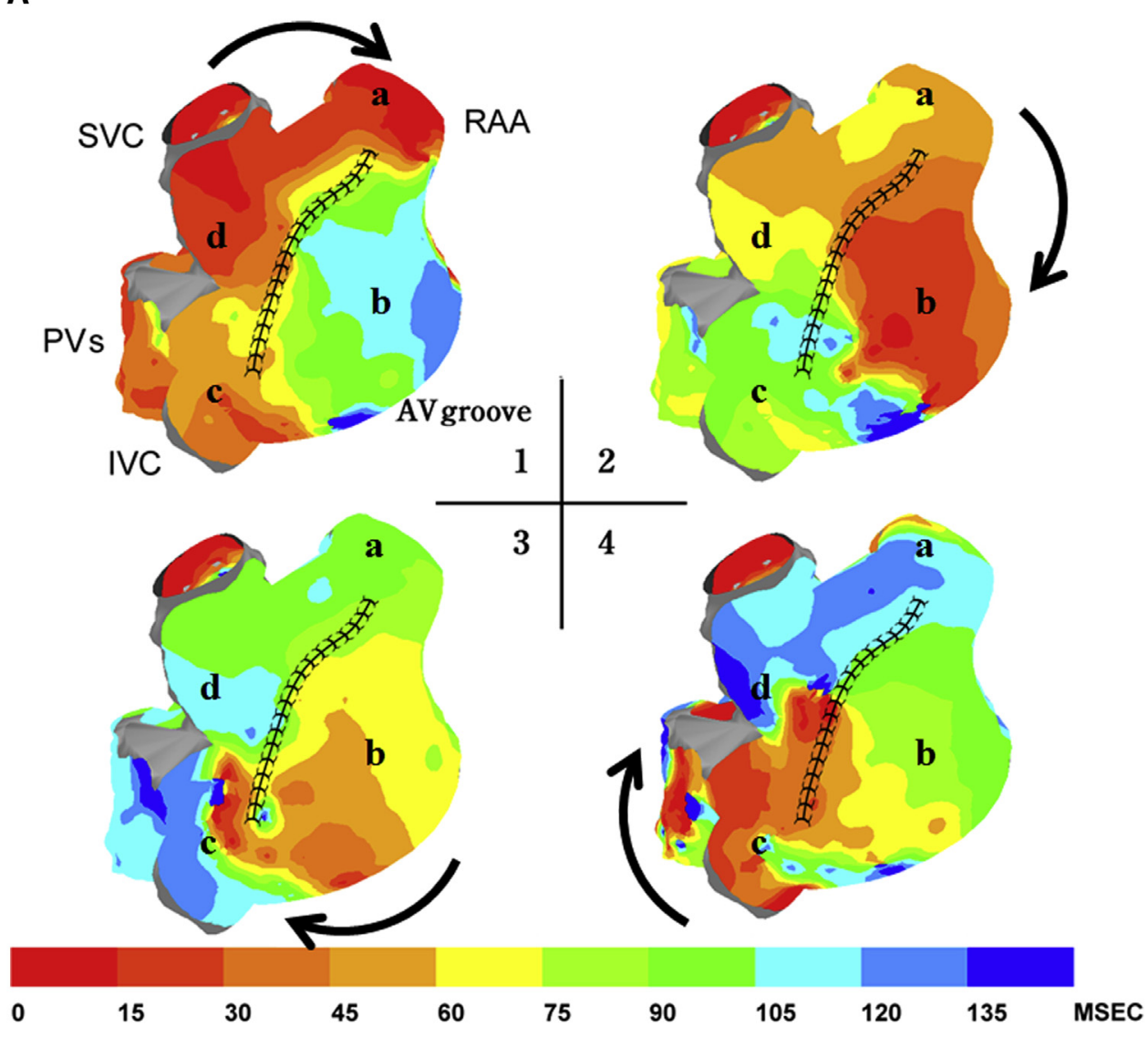

B

FIGURE 2. Atrial electrograms (A) and activation maps (B) are shown at 4 separate points in time during postoperative atrial fibrillation in the atriotomy group. The maps represent the time since the last activation, with red being the leading edge of the wavefront. The atrial activation rotates around the right atriotomy during the induced postoperative atrial fibrillation (black arrows). The maps represent the lateral right atrium. The black line represents the incision on the lateral right atrium. The location of the selected electrograms corresponds to the activation time at the 4 sites shown in (A). The time since the last activation at each time point is shown with color coding at $15-\mathrm{ms}$ increments. $E C G$, Electrocardiogram; $S V C$, superior vena cava; $R A A$, right atrial appendage; $P V s$, pulmonary veins $I V C$, inferior vena cava; $A V$ groove, atrioventricular groove. 
Activation map of induced sustained AF. Sustained AF ( $>2$ minutes) was induced only in the atriotomy group $(\mathrm{n}=5)$. Almost all of sustained AF originated from the right atrium. Three of these animals had an incisional reentrant tachycardia around the right atriotomy (Figure 2, Video 1) and 2 had a focal activation arising from the RA during AF. The LA activations in these animals were passive from the RA activation. One animal simultaneously had both an incisional reentrant tachycardia and a pulmonary vein focal activation. The cycle lengths of the incisional reentrant tachycardias and pulmonary vein focal activation, which were measured for 5 different beats in each animal, were $141 \pm 16$ and $122 \pm 19 \mathrm{~ms}$, respectively.

Activation pattern of the RA and LA during sinus rhythm (SR). Because there was no slow conduction in the activation of the anesthesia group, the RA atrial activation spread homogeneously down to the right atrial free wall from the pacing site (Figure 3). The RA activation in the atriotomy group exhibited severe inhomogeneity. The activation of the steroid group spread homogeneously parallel to the atrial incision. Although the RA activation patterns differed among the 3 groups, all LA activation in each group was homogeneous.

Inhomogeneity index of the atrial conduction during SR. The variation coefficient of the phase difference (inhomogeneity index) in the RA and LA was compared between all groups (Figure 4, A). The RA inhomogeneity index
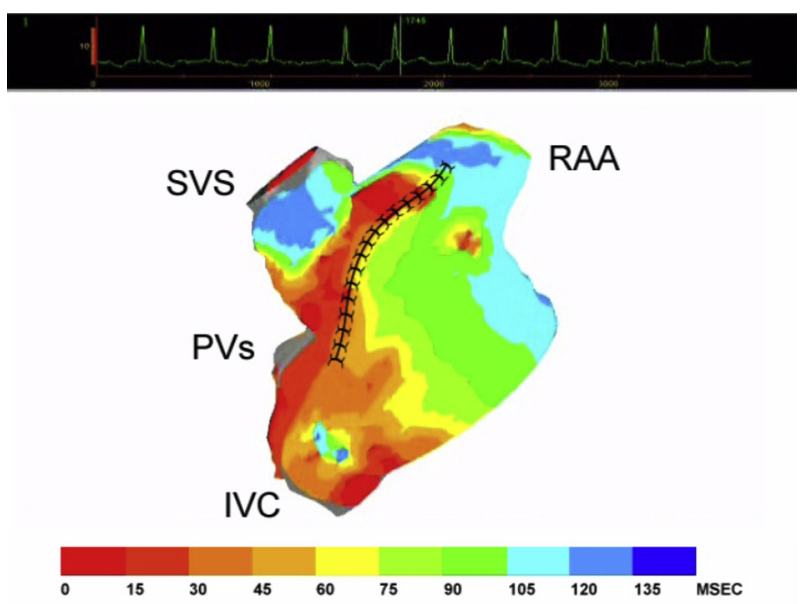

VIDEO 1. Atrial activation rotates around the right atriotomy during postoperative atrial fibrillation in a clockwise fashion in the atriotomy group. The maps represent the time since the last activation, with red being the leading edge of the wavefront. The atrial activation rotates around the right atriotomy during induced postoperative atrial fibrillation. The maps represent the lateral right atrium. The black line represents the incision on the lateral right atrium. The time since the last activation at each time point is shown with color coding at $15-\mathrm{ms}$ increments. $S V C$, Superior vena cava; $R A A$, right atrial appendage; $P V s$, pulmonary veins; $I V C$, inferior vena cava. Video available at: http://www.jtcvsonline.org/article/S00225223(17)30181-2/addons. was $1.0 \pm 0.1$ in the anesthesia group. The RA inhomogeneity index of the atriotomy group was significantly higher than that of the anesthesia group $(2.0 \pm 0.2, P<.001)$. Antiinflammatory therapy (steroid group) significantly decreased the RA inhomogeneity index after the atriotomy back toward the level in the control state (anesthesia group, $1.2 \pm 0.1, P<.001)$. The inhomogeneity index of the steroid group did not significantly differ compared with that of the anesthesia group $(P=.921)$.

The LA inhomogeneity indices of the anesthesia, atriotomy, and steroid groups were $1.2 \pm 0.2,1.4 \pm 0.5$, $1.1 \pm 0.2$, respectively. Although there was a trend toward the atriotomy group having a higher LA inhomogeneity index than the other groups, there were no significant differences between each group $(P=.231)$.

\section{Degree of Atrial Inflammation}

Myeloperoxidase activity. The myeloperoxidase activity in the RA and LA myocardium is shown in Figure 4, $B$. The RA myeloperoxidase activity in the atriotomy group was significantly higher than that of the anesthesia group $(0.7 \pm 0.2$ vs $0.2 \pm 0.1 \Delta \mathrm{OD} / \mathrm{min} / \mathrm{mg}$ protein, $P<.001)$. Anti-inflammatory therapy (steroid group) prevented atrial inflammation in the RA after the atriotomy $(0.2 \pm 0.1$ $\Delta \mathrm{OD} / \mathrm{min} / \mathrm{mg}$ protein, $P<.001)$. The LA myeloperoxidase activity of the anesthesia, atriotomy, and steroid groups was $0.1 \pm 0.1,0.2 \pm 0.2$, and $0.2 \pm 0.1$, respectively. There were no significant differences between each group $(P=.18)$. In the atriotomy group, the LA myeloperoxidase activity was significantly lower than that in the RA $(P<.001)$. Histological findings. The neutrophil cell infiltration of the RA wall in the anesthesia group was low; intensity (grade 0-2) and extent (grade 0-2). The RA tissue in the atriotomy group had more severe neutrophil cell infiltration than that in the anesthesia group. Neutrophil cells in the atriotomy group were significantly scattered in the whole RA wall; intensity (grade 3-4) and extent (grade 3-4). The RA neutrophil cell infiltration indices of the anesthesia group and the atriotomy group were $1 \pm 1$ and $14 \pm 3$, respectively $(P<.001)$. The RA neutrophil cell infiltration index of the steroid group $(2 \pm 2)$ was significantly decreased compared with the atriotomy group $(P<.001)$. There were significant differences among the 3 groups, respectively $(P<.01)$. The LA neutrophil infiltration indices of the anesthesia, atriotomy, and steroid groups were low $(1 \pm 1,2 \pm 2$, and $1 \pm 1)$. There were no significant differences between each group $(P=.08)$. The RA neutrophil cell index in the atriotomy group was significantly higher than LA neutrophil cell index $(P<.001)$.

\section{Relationship Between the Inhomogeneity of the Atrial Conduction and Degree of Atrial Inflammation}

The relationship between the inhomogeneity of the atrial conduction and degree of atrial inflammation in all groups is 
RA
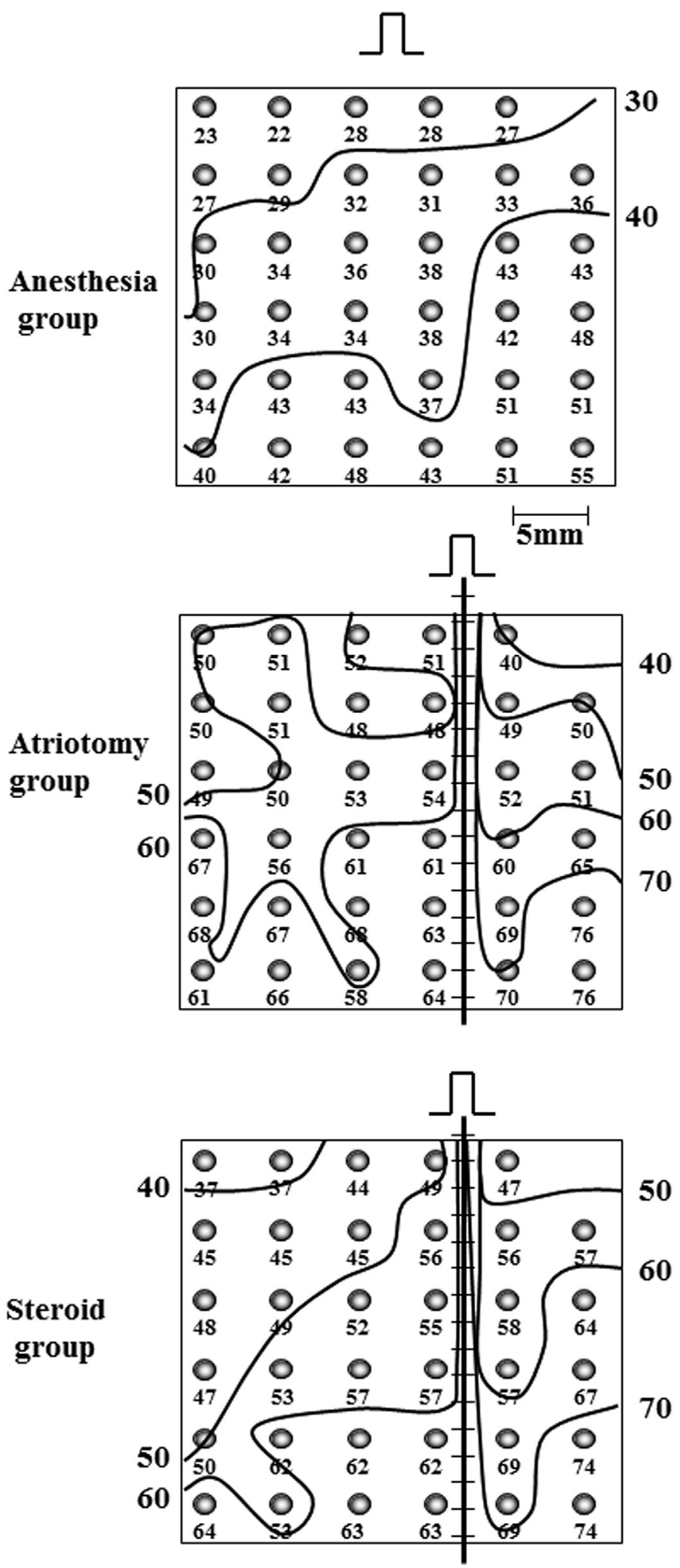

LA
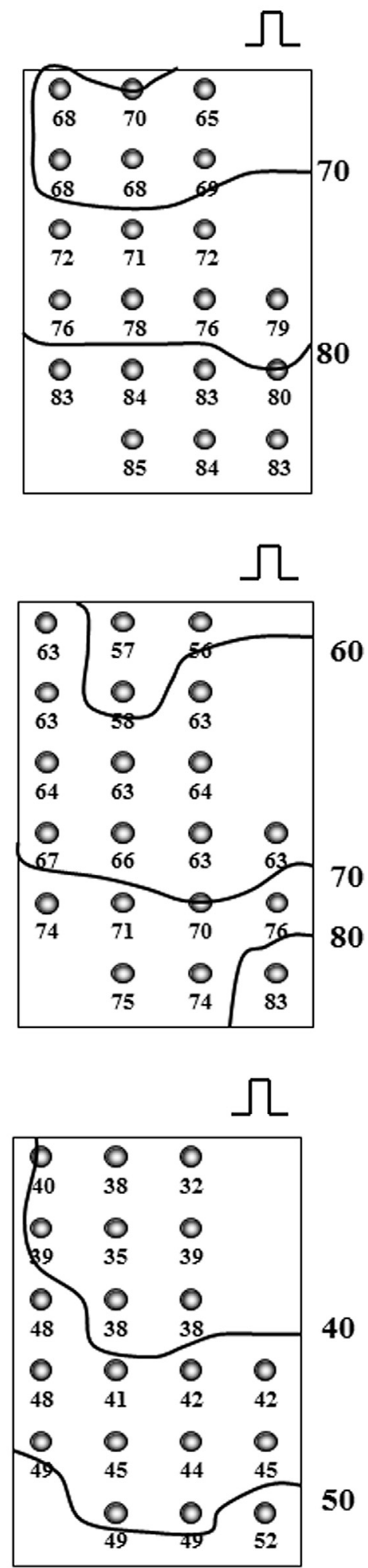

FIGURE 3. Activation maps from selected regions of the right atrium $(R A)$ and left atrium $(L A)$ during atrial pacing. Typical examples of activation maps are shown from each group. Note that the inhomogeneity of the conduction increased from the anesthesia group to the atriotomy group in the RA. The steroid group normalized to the control levels. The LA activations in the anesthesia, atriotomy, and steroid groups are homogeneous. The rectangle indicates the pacing site. The vertical line in the center of the panel in the atriotomy and steroid groups indicates the atrial incision line. 

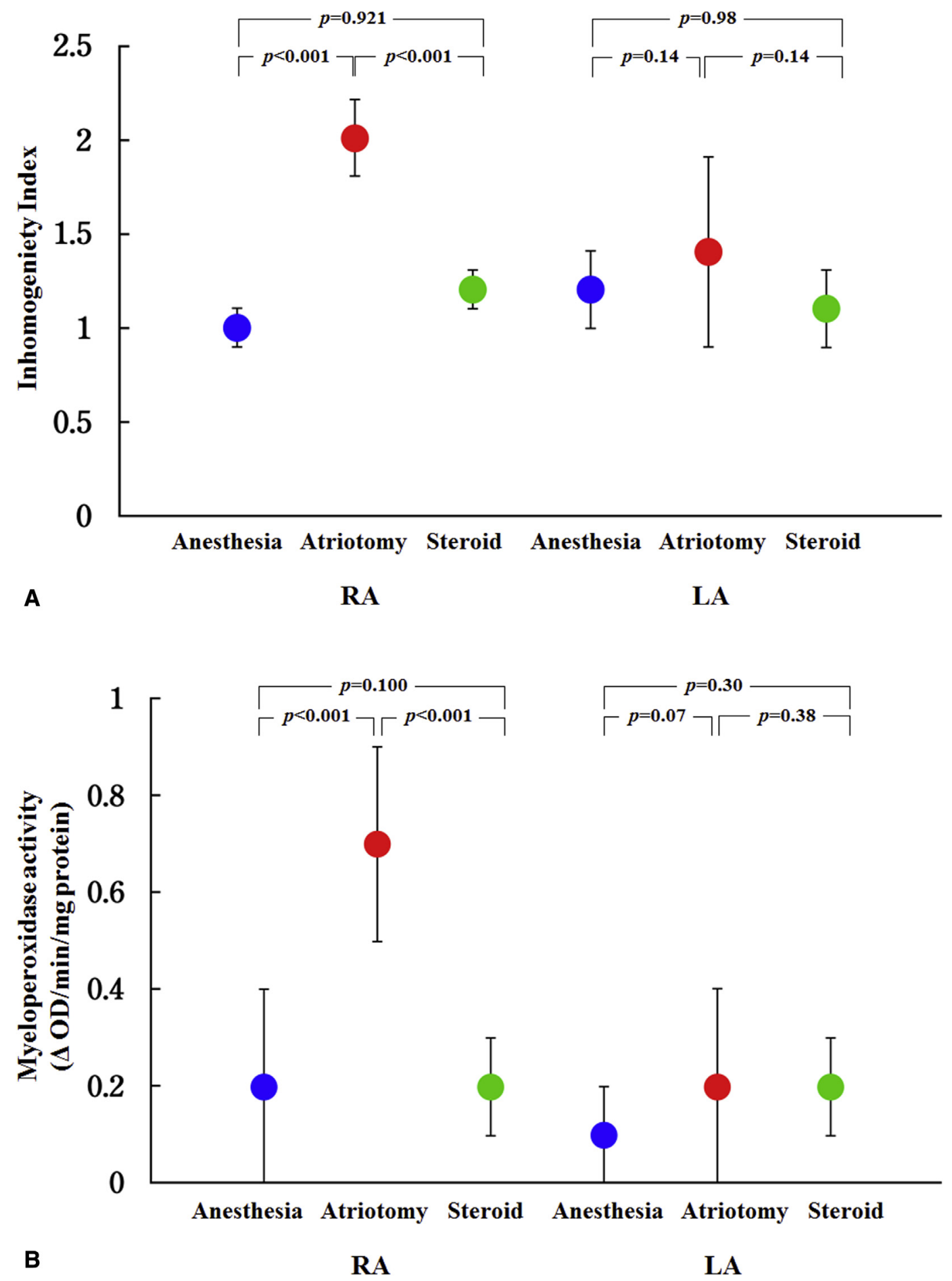

FIGURE 4. A, Inhomogeneity indices of the right atrium $(R A)$ and left atrium $(L A)$ conduction. B, Myeloperoxidase activity of the RA and LA myocardium. $O D$, Optical density.

shown in Figure 5. The inhomogeneity of the atrial conduction correlated closely with the myeloperoxidase activity $(r=0.51, P=.03)$ and neutrophil cell infiltration index $(r=0.44, P=.07)$. The duration of induced AF significantly correlated with the degree of RA inhomogeneity $(r=0.75, P<.001)$, RA myeloperoxidase activity $(r=0.74, P<.001)$, and RA neutrophil cell index $(r=0.80, P<.001)$.

\section{DISCUSSION}

This experimental study showed that reentrant circuits responsible for $\mathrm{AF}$ are dependent on the degree of 


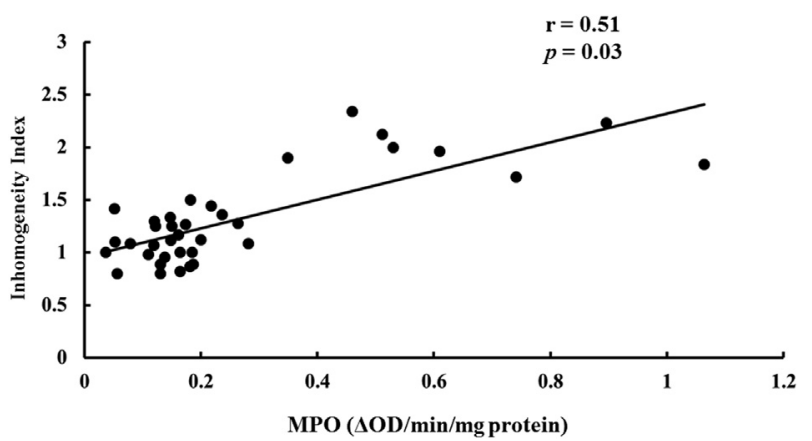

FIGURE 5. Relationship between the inhomogeneity of the atrial conduction and myeloperoxidase $(M P O)$ activity. The inhomogeneity of the atrial conduction is associated with a proportional increase in the MPO activity $(r=0.51, P=.03)$. The line indicates the approximate line of data. $O D$, Optical density.

inflammation and occur in the region of greatest inflammation, inhomogeneous conduction, and the atriotomy. Antiinflammatory therapy in this study eliminated the risk of POAF.

In this study, the degree of inhomogeneity of the atrial conduction significantly correlated with the incidence and duration of induced AF. Studies have shown that inhomogeneous atrial conduction is associated with the initiation of AF. ${ }^{14,19}$ Spatial inhomogeneity of conduction can cause local conduction block or slow conduction, resulting in the initiation of AF. The inhomogeneity of the atrial conduction significantly differed between the RA and LA in this study. The RA had more inhomogeneous atrial conduction compared with the LA. The mapping data showed that the inhomogeneous atrial conduction around the atriotomy developed reentrant circuits or focal activation, which facilitated AF. Spatial inhomogeneity of the atrial conduction could cause POAF.

Most POAF following cardiac surgery appears within the first 3 PODs and resolves within 3 weeks. ${ }^{1-3}$ Patients following the Maze procedure also have POAF temporarily, despite their pulmonary veins having been completely isolated to block any focal activation from the pulmonary veins, and the atrium was incised to block macroreentry. ${ }^{20}$ The specific substrates responsible for POAF following cardiac surgery may differ from those of chronic AF. In general, although it has been reported that $\mathrm{AF}$ begets $\mathrm{AF},{ }^{21}$ POAF disappears naturally without any antiarrhythmic medication. One possible mechanism of POAF is increased dispersion of atrial refractoriness. ${ }^{22} \mathrm{Ab}-$ normalities in the atrial conduction and repolarization have both been shown to be important substrates for AF. Some studies have shown that an increased inflammatory response correlates with the occurrence of POAF. It has been shown that an elevated C-reactive protein is associated with the occurrence of POAF. ${ }^{11,12}$ Bruins and colleagues ${ }^{10}$ also have shown that the incidence of AF is associated with serial changes in inflammation following cardiac surgery.
These data suggest that the reduction of atrial inflammation during the wound-healing process may account for the termination of the POAF. Our data would support this hypothesis.

The incidence of induced POAF in the present study was $100 \%$ in the atriotomy group. Continuous AF was also observed in only the atriotomy group. Antiinflammatory therapy eliminated the induced POAF after the atriotomy. The histological findings of the present study showed that the neutrophil cell infiltration was scattered in the entire RA wall around the atrial incision. Because the atrial wall is very thin, the inflamed atrium tissue around the atrial incision might affect the atrial conduction properties. This study focused on the neutrophil cells in the atrial wall. However, there might have been significant edema in the atrial wall in the atriotomy group. Exposing individual cells to activated neutrophils might alter the action potential duration and cause spontaneous arrhythmias. The platelet activation factor (PAF) released from neutrophil cells exerts arrhythmogenic effects. It has been described that PAF causes a consistent and dose-dependent set of alternations in the transmembrane potentials, including an increased action potential duration and runs of early after depolarizations. ${ }^{23}$ Inflammation is a complex process participating in releasing cytokines or increased oxidative stress. Proinflammatory cytokines, such as interleukin or tumor necrosis factor (TNF)- $\alpha$, also influence the development of POAF. Interleukin-6 levels are significantly associated with the incidence of POAF. $^{24}$ It has been demonstrated that interleukin- 6 or TNF- $\alpha$ decrease calcium currents in the myocytes, ${ }^{25}$ resulting in a reduction of the action potential duration and refractory period, and induction and maintenance of AF by multiple circuit reentry. ${ }^{26} \mathrm{TNF}-\alpha$ and oxidative stress also downregulate gap junction proteins and reduce cell coupling. ${ }^{27}$ Steroid therapy could reverse these changes.

Clinical studies have shown that anti-inflammatory therapy significantly reduces the incidence of POAF following cardiac surgery. ${ }^{28-31}$ A large randomized clinical trial about steroid therapy for POAF is now ongoing. ${ }^{32}$ The present study showed that anti-inflammatory therapy reduced the atrial inflammation after the atriotomy, resulting in a homogeneous atrial conduction and lower incidence of induced POAF, although the dosages of methylprednisolone used in the present study were adequately high enough to completely suppress the inflammatory response, and such high doses would not be acceptable for humans because of the resulting complications, such as a high risk of infection. ${ }^{33}$ Atrial inflammation was localized in the RA in the present study. Therefore, it may not be needed to systemically reduce inflammation to prevent POAF. Anti-inflammatory therapy localized to the inflamed region may be effective in preventing POAF. 


\section{Study Limitations}

This study was designed to prove the mechanisms of inducing POAF after cardiac surgery by evaluating the electrophysiology and immunopathology of the RA and LA. Further study should be performed to check for spontaneous POAF after cardiac surgery. Although the pathological study was checked by a blinded pathologist, the electrophysiological studies were not blind tests. For fairness, the electrophysiological examination protocols were exactly the same for each of the groups.

\section{Conflict of Interest Statement}

Dr Damiano reports consulting fees from Atricure, lecture fees and grant support from Edwards and On-X Lifesciences, and grant support from Thrasos Inc. Dr Gaynor is presently the chief medical officer for Atricure. All other authors have nothing to disclose with regard to commercial support.

We acknowledge the excellent technical assistance of P. Diane Toeniskoetter, Kathryn L. Cook, Naomi R. Still, Geneva R. Baca, and Dennis C. Gordon. We also thank Dawn G. Schuessler for the preparation of the manuscript.

\section{References}

1. Fuller JA, Adams GG, Buxton B. Atrial fibrillation after coronary artery bypass grafting. Is it a disorder of the elderly? J Thorac Cardiovasc Surg. 1989;97: $821-5$.

2. Creswell LL, Schuessler RB, Rosenbloom M, Cox JL. Hazards of postoperative atrial arrhythmias. Ann Thorac Surg. 1993;56:539-49.

3. Aranki SF, Shaw DP, Adams DH, Rizzo RJ, Couper GS, VanderVliet M, et al. Predictors of atrial fibrillation after coronary artery surgery: current trends and impact on hospital resources. Circulation. 1996;94:390-7.

4. Echahidi N, Pibarot P, O'Hara G, Mathieu P. Mechanisms, prevention, and treatment of atrial fibrillation after cardiac surgery. J Am Coll Cardiol. 2008;51: 793-801.

5. Mathew JP, Parks R, Savino JS. Atrial fibrillation following coronary artery bypass graft surgery: predictors, outcomes, and resource utilization. MultiCenter Study of Perioperative Ischemia Research Group. JAMA. 1996;276:300-6.

6. Kowey PR, Dalessandro DA, Herbertson R, Briggs B, Wertan MA, Rials SJ, et al. Effectiveness of digitalis with or without acebutolol in preventing atrial arrhythmias after coronary artery surgery. Am J Cardiol. 1997;79:1114-7.

7. Randomised trial of normothermic versus hypothermic coronary bypass surgery. The Warm Heart Investigators. Lancet. 1994;343:559-63.

8. Butler J, Chong JL, Rocker GM, Pillai R, Westaby S. Atrial fibrillation after coronary artery bypass grafting: a comparison of cardioplegia versus intermittent aortic cross-clamping. Eur J Cardiothorac Surg. 1993;7:23-5.

9. Almassi GH, Schowalter T, Nicolosi AC, Aggarwal A, Moritz TE, Henderson WG, et al. Atrial fibrillation after cardiac surgery. A major morbid event? Ann Surg. 1997;226:501-13.

10. Bruins P, te Velthuis H, Yazdanbakhsh AP, Jansen PG, van Hardevelt FW, de Beaumont EM, et al. Activation of the complement system during and after cardiopulmonary bypass surgery: postsurgery activation involves C-reactive protein and is associated with postoperative arrhythmia. Circulation. 1997;96:3542-8.

11. Chung MK, Martin DO, Sprecher D, Wazni O, Kanderian A, Carnes CA, et al. C-reactive protein elevation in patients with atrial arrhythmias: inflammatory mechanisms and persistence of atrial fibrillation. Circulation. 2001;104:2886-91.
12. Aviles RJ, Martin DO, Apperson-Hansen C, Houghtaling PL, Rautaharju P, Kronmal RA, et al. Inflammation as a risk factor for atrial fibrillation. Circulation. 2003;108:3006-10.

13. Jacob KA, Nathoe HM, Dieleman JM, van Osch D, Kluin J, van Dijk D. Inflammation in new-onset atrial fibrillation after cardiac surgery: a systematic review. Eur J Clin Invest. 2014;44:402-28.

14. Ishii Y, Schuessler RB, Gaynor SL, Yamada K, Fu AS, Boineau JP, et al. Inflammation of the atrium after cardiac surgery is associated with inhomogeneity of atrial conduction and atrial fibrillation. Circulation. 2005;111:2881-8.

15. Lee R, Nitta T, Schuessler RB, Johnson DC, Boineau JP, Cox JL. The closed heart MAZE: a nonbypass surgical technique. Ann Thorac Surg. 1999;67:1696-702.

16. Lammers WJ, Schalij MJ, Kirchhof CJ, Allessie MA. Quantification of spatial inhomogeneity in conduction and initiation of reentrant atrial arrhythmias. Am J Physiol. 1990;259:H1254-63.

17. Krawisz JE, Sharon P, Stenson WF. Quantitative assay for acute intestinal inflammation based on myeloperoxidase activity. Assessment of inflammation in rat and hamster models. Gastroenterology. 1984;87:1344-50.

18. Mehta JL, Nichols WW, Schofield R, Donnelly WH, Chandna VK. TxA2 inhibition and ischemia-induced loss of myocardial function and reactive hyperemia. Am J Physiol. 1990;258:H1402-8.

19. Wang Z, Feng J, Nattel S. Idiopathic atrial fibrillation in dogs: electrophysiologic determinants and mechanisms of antiarrhythmic action of flecainide. J Am Coll Cardiol. 1995;26:277-86.

20. Ishii Y, Gleva MJ, Gamache MC, Schuessler RB, Boineau JP, Bailey MS, et al Atrial tachyarrhythmias after the maze procedure: incidence and prognosis. Circulation. 2004;110:II164-8.

21. Wijffels MC, Kirchhof CJ, Dorland R, Allessie MA. Atrial fibrillation begets atrial fibrillation. A study in awake chronically instrumented goats. Circulation. 1995;92:1954-68.

22. Cox JL. A perspective of postoperative atrial fibrillation in cardiac operations Ann Thorac Surg. 1993;56:405-9.

23. Hoffman BF, Guo SD, Feinmark SJ. Arrhythmias caused by platelet activating factor. J Cardiovasc Electrophysiol. 1996;7:120-33.

24. Gaudino M, Andreotti F, Zamparelli R, Di Castelnuovo A, Nasso G, Burzotta F, et al. The $-174 \mathrm{G} / \mathrm{C}$ interleukin-6 polymorphism influences postoperative interleukin-6 levels and postoperative atrial fibrillation. Is atrial fibrillation an inflammatory complication? Circulation. 2003;108:II195-9.

25. Sugishita K, Kinugawa K, Shimizu T, Harada K, Matsui H, Takahashi T, et al Cellular basis for the acute inhibitory effects of IL-6 and TNF- alpha on excitation-contraction coupling. J Mol Cell Cardiol. 1999;31:1457-67.

26. Nattel S. New ideas about atrial fibrillation 50 years on. Nature. 2002;415 219-26.

27. Fernandez-Cobo M, Gingalewski C, Drujan D, De Maio A. Downregulation of connexin 43 gene expression in rat heart during inflammation. The role of tumour necrosis factor. Cytokine. 1999;11:216-24.

28. Yared JP, Starr NJ, Torres FK, Bashour CA, Bourdakos G, Piedmonte M, et al. Effects of single dose, postinduction dexamethasone on recovery after cardiac surgery. Ann Thorac Surg. 2000;69:1420-4.

29. Mathew JP, Fontes ML, Tudor IC, Ramsay J, Duke P, Mazer CD, et al. A multicenter risk index for atrial fibrillation after cardiac surgery. JAMA. 2004;291: 1720-9.

30. Rezaei Y. Anti-inflammatory role of statins in preventing postoperative atrial fibrillation. Am J Cardiol. 2016;117:314.

31. Raiten JM, Ghadimi K, Augoustides JG, Ramakrishna H, Patel PA, Weiss SJ, et al. Atrial fibrillation after cardiac surgery: clinical update on mechanisms and prophylactic strategies. J Cardiothorac Vasc Anesth. 2015;29:806-16.

32. Whitlock R, Teoh K, Vincent J, Devereaux PJ, Lamy A, Paparella D, et al. Rationale and design of the steroids in cardiac surgery trial. Am Heart J. 2014;167: 660-5.

33. Marik PE, Fromm R. The efficacy and dosage effect of corticosteroids for the prevention of atrial fibrillation after cardiac surgery: a systematic review. J Crit Care. 2009;24:458-63.

Key Words: fibrillation, inflammation, surgery 